\title{
Center Vortices at Strong Couplings
}

\author{
M. Faber ${ }^{\mathrm{a}}$, J. Greensite ${ }^{\mathrm{b}}$, Š. Olejník ${ }^{\mathrm{c}}$ \\ ${ }^{\text {a} I n s t . ~ f u ̈ r ~ K e r n p h y s i k, ~ T e c h . ~ U n i v . ~ W i e n, ~ A-1040 ~ V i e n n a, ~ A u s t r i a ~}$

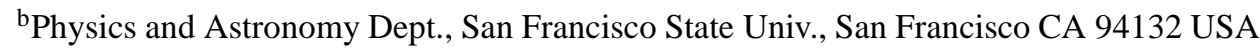 \\ ${ }^{\mathrm{c} I n s t . ~ o f ~ P h y s ., ~ S l o v a k ~ A c a d . ~ o f ~ S c i ., ~ S K-842 ~} 28$ Bratislava, Slovakia
}

A long-range effective action is derived for strong-coupling lattice $\mathrm{SU}(2)$ gauge theory in $D=3$ dimensions. It is shown that center vortices emerge as stable saddlepoints of this action.

The behavior of Wilson loops $W_{r}(C)$ in higher group representations $r$ is an important clue about the nature of the confining force. It is well known that the asymptotic string tension $\sigma_{r}$, in $\mathrm{SU}(N)$ gauge theory, depends only on the $N$-ality of the representation $r$. This follows from a simple energetics argument based on the color screening of an external charge by gluons. On the other hand, $W_{r}(C)$ is a vacuum expectation value, and it therefore carries information about the probability distribution of vacuum fluctuations in the absence of external sources. Since it is generally believed that Wilson loops are disordered via the positional fluctuations of some class of large-scale confining configurations, it follows that such configurations must have the very non-trivial property that $N$-ality $=0$ loops are somehow not disordered, and the asymptotic string tension induced in other loops depends only on their $\mathrm{N}$-ality. The only confining configurations known to have this property are center vortices. Confining instanton models, for example, tend to disorder all loops, regardless of $\mathrm{N}$-ality, while monopole Coulomb gas and dual superconductor models typically predict confinement of certain (double electric) charges of $N$-ality $=0$. These properties are ruled out by general arguments (screening) and by numerical data [1].

However, if the $N$-ality dependence of the string tension implies a center vortex confinement mechanism, it follows that confinement in a supposedly very well-understood case, namely, strong-coupling lattice gauge theory in $D>2$ dimensions, is also due to a vor-

\footnotetext{
*Talk presented by J. Greensite. Work supported by the U.S. Department of Energy under Grant No. DE-FG03-92ER40711.
}

tex mechanism. In $D>2$ dimensions, one can easily demonstrate from a strong-coupling expansion that, e.g., $N$-ality $=0$ loops have an asymptotic perimeterlaw falloff. One can, in fact, go further, and show at strong couplings that confining disorder in $D>2$ dimension is due to to center disorder, rather than plaquette disorder [2]: Consider a very large planar loop $C$, and let its minimal area be subdivided into many sub-areas bounded by large loops $C_{i}$. The question is whether the holonomies $U\left(C_{i}\right)$ are uncorrelated in $D>2$ dimensions, as they are in $D=2$ dimensions. The test is whether, in general,

$$
\left\langle\prod_{i} F\left[U\left(C_{i}\right)\right]\right\rangle \stackrel{?}{=} \prod_{i}\left\langle F\left[U\left(C_{i}\right)\right]\right\rangle
$$

where $F[g]$ is any class function with $\int d g F[g]=0$. The equality is satisfied in $D=2$ dimensions, but in any higher dimension it can be shown that

$$
\left\langle\prod_{i} F\left[U\left(C_{i}\right)\right]\right\rangle \gg \prod_{i}\left\langle F\left[U\left(C_{i}\right)\right]\right\rangle
$$

which means that the holonomies $U\left(C_{i}\right)$ are in fact correlated in some way. On the other hand, if $z[g] \in$ $Z_{N}$ is the center element closest to $g$ on the group manifold, one finds

$$
\left\langle\prod_{i} z\left[U\left(C_{i}\right)\right]\right\rangle \approx \prod_{i}\left\langle z\left[U\left(C_{i}\right)\right]\right\rangle
$$

to leading order in $\beta$. In other words, the coset elements in $S U(N) / Z_{N}$ associated with $U\left(C_{i}\right)$ holonomies are correlated; it is the center elements $z\left[U\left(C_{i}\right)\right] \in Z_{N}$ which are (nearly) uncorrelated [2], and which give rise to the area law falloff. 


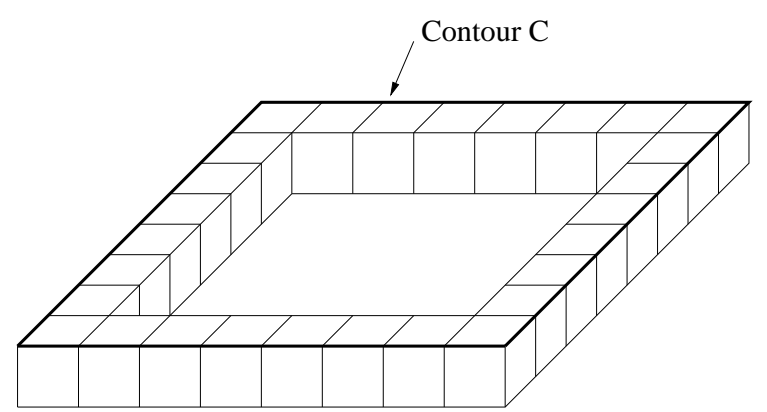

Figure 1. Arrangement of $U$-plaquettes in a tube around a rectangular contour $C$ on the coarse $V$ lattice.

The fact that confining disorder is essentially center disorder provides some evidence in favor of a vortex mechanism in strong-coupling lattice gauge theory. But we would still like to understand more explicitly how vortices arise in this theory. Although center vortices are certainly not stable saddlepoints of the Wilson action, it is possible that they might be stable saddlepoints of some long-range effective action. Vortex structure in the vacuum should become apparent at length scales greater than the vortex thickness, and this thickness is expected [3] to be on the order of the adjoint string-breaking length, which is approximately four lattice units for $\beta / 4 \ll 1$. With this in mind, we define an effective action $S_{\text {eff }}$ on a coarse lattice, derived by integrating out links on a finer lattice, via

$$
\begin{aligned}
& \exp \left[S_{\text {eff }}[V]\right] \equiv \\
& \left.\quad \int D U \prod_{l^{\prime}} \delta\left[V_{l^{\prime}}^{\dagger}(U U . . U)_{l^{\prime}}\right)-I\right] e^{S_{W}[U]}
\end{aligned}
$$

The length of links on the coarse $V$-lattice is equal to $L$ lattice spacings on the original $U$-lattice. It is not hard to see that $S_{\text {eff }}$ is non-local in $D>2$ dimensions. For $\mathrm{SU}(2)$ gauge theory, $S_{\text {eff }}$ will contain large loops in all $j=$ integer representations with only perimeter-falloff coefficients. These terms are derived from "tube" diagrams, as shown in Fig. 1. leading to contributions such as

$$
\begin{gathered}
\left.\exp \left[S_{e f f}[V]\right] \supset \int D U_{l \in C} \prod_{l^{\prime} \in C} \delta\left[V_{l^{\prime}}^{\dagger}(U U . . U)_{l^{\prime}}\right)-I\right] \\
\times\left(\frac{\beta}{4}\right)^{4(P(C)-4)}(\operatorname{Tr}[U(C)])^{2}
\end{gathered}
$$

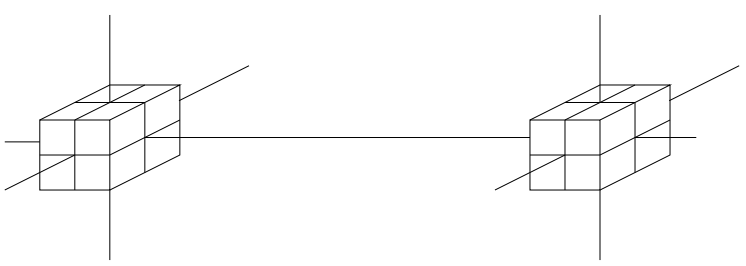

Figure 2. The degrees of freedom in $\tilde{S}_{L}[V, \tilde{U}]$.

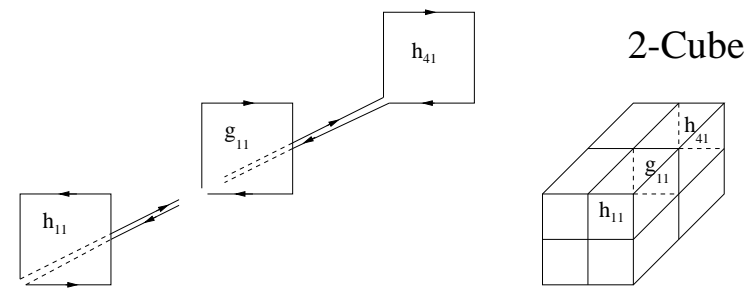

Figure 3. Three plaquette variables on the 2-cube.

$$
\supset\left(\frac{\beta}{4}\right)^{4(P(C)-4)} \chi_{1}[V(C)]
$$

We would like to derive, instead, an effective action where the leading contributions to any Wilson loop on the $V$-lattice (including $j=$ integer loops) are obtained from local terms. To achieve this, working in $D=3$ dimensions, we consider integrating (in eq. (4)) all links on the the $U$-lattice except those belonging to cubes of volume $2^{3}$ centered at sites on the $V$-lattice, as shown in Fig. 2. This procedure defines the effective action $\tilde{S}_{L}$

$$
\begin{aligned}
Z= & \int D V \int \prod_{l \in 2-\text { cubes }} d \tilde{U}_{l} \int \prod_{l^{\prime \prime} \notin 2-\text { cubes }} d U_{l^{\prime \prime}} \\
& \left.\prod_{l^{\prime}} \delta\left[V_{l^{\prime}}^{\dagger}(U U . . U)_{l^{\prime}}\right)-I\right] e^{S_{W}[U]} \\
\equiv & \int D V \int \prod_{l \in 2-\text { cubes }} d \tilde{U}_{l} \exp \left[\tilde{S}_{L}[V, \tilde{U}]\right]
\end{aligned}
$$

Introduce group-valued plaquette variables $h, g$ lying in the 2-cubes centered at $V$-lattice sites, with $h$ variables running around plaquettes on the surface of the 2-cube, and $g$ variables running around plaquettes in the interior, as indicated in Fig. 3. After integrating over $U$-links not in 2-cubes, we find

$Z \approx \int D V D \tilde{U} \exp \left[\frac{\beta}{2} \sum(\operatorname{Tr}[h]+\operatorname{Tr}[g])\right.$ 


$$
\begin{aligned}
& +2\left(\frac{\beta}{4}\right)^{4(L-2)} \sum_{l^{\prime}} f_{l^{\prime}}^{i j k l} \operatorname{Tr}\left[h_{i j}^{\dagger} V_{l^{\prime}} h_{k l}^{\dagger} V_{l^{\prime}}^{\dagger}\right] \\
& \left.+2\left(\frac{\beta}{4}\right)^{L^{2}-4} \sum_{P^{\prime}} \operatorname{Tr}\left[V g V g V^{\dagger} g^{\dagger} V^{\dagger} g^{\dagger}\right]\right]
\end{aligned}
$$

Now change integration variables from $\tilde{U}$ links to plaquette variables $h, g$ on 2-cubes. This introduces a Bianchi constraint on each 1-cube [4]. Symbolically,

$$
\begin{aligned}
\delta[\text { Bianchi }] & =\delta[\text { hghghg }-I] \\
& =\sum_{j=0, \frac{1}{2}, 1, . .}(2 j+1) \chi_{j}[\text { hghghg }]
\end{aligned}
$$

After integrating over the $g$-variables, we obtain (writing only terms of low order in $\beta$ and $h$ )

$$
\begin{aligned}
Z \approx & \int D V D h \prod_{2-\text { cubes } K} \\
& \left\{1+2\left(\frac{\beta}{4}\right)^{3} \sum_{c \in K} \chi_{\frac{1}{2}}\left[(h h h)_{c}\right]\right. \\
& \left.+2\left(\frac{\beta}{4}\right)^{4} \sum_{a d j a c e n t} \chi_{\frac{1}{2}}\left[(h h h)_{c_{1}}(h h h)_{c_{2}}\right]+\ldots\right\} \\
\times \quad & \exp \left[\frac{\beta}{2} \sum \operatorname{Tr}[h]\right. \\
& +2\left(\frac{\beta}{4}\right)^{4(L-2)} \sum_{l^{\prime}} f_{l^{\prime}}^{i j k l} \operatorname{Tr}\left[h_{i j}^{\dagger} V_{l^{\prime}} h_{k l}^{\dagger} V_{l^{\prime}}^{\dagger}\right] \\
& \left.+2\left(\frac{\beta}{4}\right)^{L^{2}} \sum_{P^{\prime}} \operatorname{Tr}\left[V V V^{\dagger} V^{\dagger}\right]\right] \\
\approx & \int D V D h \exp \left[S_{L}[V, h]\right]
\end{aligned}
$$

This resembles an adjoint-Higgs theory, with an $\mathrm{SU}(2)$ gauge field $V_{\mu}$ coupled to 24 "matter" fields $h$ in the adjoint representation. Note that for coarse lattices (large $L$ ), the "Higgs" potential term is much larger than the "kinetic" and pure-gauge ( $V$-plaquette) terms, so the $h$-fields fluctuate almost independent of $V_{\mu}$.

Next we fix some of the $h$-fields by a choice of unitary gauge, leaving a remnant $Z_{2}$ gauge invariance, and integrate out the remaining $h$ d.o.f. to find

$S_{\text {eff }}[V] \approx S_{\text {link }}\left[V,\langle h\rangle_{h}\right]+S_{\text {plaq }}[V]$

$$
\begin{aligned}
& =2\left(\frac{\beta}{4}\right)^{4(L-2)} \sum_{l^{\prime}} f_{l^{\prime}}^{i j k l} \operatorname{Tr}\left[\left\langle h_{i j}^{\dagger}\right\rangle_{h} V_{l^{\prime}}\left\langle h_{k l}^{\dagger}\right\rangle_{h} V_{l^{\prime}}^{\dagger}\right] \\
& +2\left(\frac{\beta}{4}\right)^{L^{2}} \sum_{P^{\prime}} \operatorname{Tr}\left[V V V^{\dagger} V^{\dagger}\right]
\end{aligned}
$$

where the expectation value of the plaquette variable $h_{i j}$ is determined almost entirely, at large $L$ and small $\beta$, by the gauge-fixed "Higgs potential" term alone (c.f. [5] for details).

We are now ready to look for saddlepoints of this effective action. We find that $S_{\text {link }}$ is maximized at

$$
\begin{aligned}
V_{\mu}(\vec{n}) & =Z_{\mu}(\vec{n}) \times g(\vec{n}) g^{\dagger}(\vec{n}+\mu) \\
Z_{\mu} & = \pm 1
\end{aligned}
$$

where $g(\vec{n}) g^{\dagger}(\vec{n}+\mu)$ is fixed by the particular unitary gauge choice, while $S_{\text {plaq }}$ is maximized if $Z Z Z Z=$ +1 . This is the unitary gauge ground state.

Consider a thin center vortex created on this state by a discontinuous gauge transformation, e.g.

$$
\begin{aligned}
& Z_{y}(\vec{n})= \begin{cases}-1 & n_{1} \geq 2, n_{2}=1 \\
+1 & \text { otherwise }\end{cases} \\
& Z_{x}(\vec{n})=Z_{z}(\vec{n})=1
\end{aligned}
$$

This configuration is stationary: $S_{\text {link }}[V]$ is still a maximum, $S_{\text {plaq }}$ is extremal (max or min) on all plaquettes. Stability depends on the eigenvalues of

$$
\begin{aligned}
& \frac{\delta^{2} S_{\text {eff }}}{\delta V_{\mu}\left(n_{1}\right) \delta V_{v}\left(n_{2}\right)}= \\
& \quad \frac{\delta^{2} S_{\text {link }}}{\delta V_{\mu}\left(n_{1}\right) \delta V_{v}\left(n_{2}\right)}+\frac{\delta^{2} S_{\text {plaq }}}{\delta V_{\mu}\left(n_{1}\right) \delta V_{v}\left(n_{2}\right)}
\end{aligned}
$$

and we find

$$
\begin{aligned}
\frac{\delta^{2} S_{\text {link }}}{\delta V_{\mu}\left(n_{1}\right) \delta V_{v}\left(n_{2}\right)} & \sim\left(\frac{\beta}{4}\right)^{4(L-2)+12} \\
\frac{\delta^{2} S_{\text {plaq }}}{\delta V_{\mu}\left(n_{1}\right) \delta V_{v}\left(n_{2}\right)} & \sim\left(\frac{\beta}{4}\right)^{L^{2}}
\end{aligned}
$$

The crucial observation is that for $\beta / 4 \ll 1$ and

$$
4(L-2)+12<L^{2} \Longrightarrow L \geq 5
$$

the contribution of $\delta^{2} S_{\text {plaq }} / \delta V \delta V$ to the stability matrix (and therefore to the eigenvalues of the stability matrix) is negligible compared to $\delta^{2} S_{\text {link }} / \delta V \delta V$, which implies 
- Vortex Stability: The thin vortex is a stable saddlepoint of the full effective action $S_{\text {eff }}$ at $L \geq 5$.

- Vortex Thickness: A "thin" vortex on the $V$ lattice means thickness less than $L$ on the $U$ lattice. This means that stable center vortices are $\approx 4-5$ lattice spacings thick. As it happens, this is also the distance where the adjoint string breaks. The correspondence between the adjoint string-breaking scale, and the thickness of center vortices, was suggested by us some years ago, in ref. [3], in connection with Casimir scaling of adjoint loops.

- Percolation: From $S_{e f f}$, we see that center vortices in $\mathrm{D}=3$ dimensions cost, in action,

$8\left(\frac{\beta}{4}\right)^{L^{2}} /$ unit length

while the entropy is $\mathrm{O}(1) /$ unit length. Since vortex entropy exceeds vortex action, these configurations percolate through the lattice, and confine $N$-ality $\neq 0$ charge.

We conclude that even in strong-coupling lattice gauge theory, the asymptotic string tension is due to the disordering effect of center vortices. Details of the analysis presented here can be found in ref. [5].

\section{REFERENCES}

1. J. Ambjørn, J. Giedt, J. Greensite, JHEP 0002 (2000) 033, hep-lat/9907021.

2. J. Ambjørn and J. Greensite, JHEP 9805 (1998) 004, hep-lat/9804022.

3. M. Faber, J. Greensite, and Š. Olejník, Phys. Rev. D57 (1998) 2603, hep-lat/9710039,

M. Faber, J. Greensite, and S. Olejník, Acta Physica Slovaca 49 (1999) 177, hep-lat/9807008.

4. G. Batrouni, Nucl. Phys. B208 (1982) 467.

5. M. Faber, J. Greensite, and Š. Olejník, JHEP 0006 (2000) 041, hep-lat/0005017. 